\title{
Uma análise cognitiva entre a emergência de padrões em narrativas infantis e elementos do Pensamento Computacional
}

\author{
Fernanda Pires ${ }^{1,2}$, José Carlos Duarte ${ }^{1}$, Larissa Pessoa ${ }^{2}$, Karla Susiane Pereira ${ }^{2}$, \\ Rafaela Ferreira $^{1}$, Rosiane de Freitas ${ }^{2}$ \\ ${ }^{1}$ Escola Superior de Tecnologia - Universidade do Estado do Amazonas (UEA) \\ ${ }^{2}$ Instituto de Computação - Universidade Federal do Amazonas (UFAM) \\ \{fpires, jcsdf.inf, rmf.lic16\}@uea.edu.br , \{lsp, karla.pereira, rosiane\}icomp.ufam.edu.br,
}

\begin{abstract}
Much has been said about the importance of the development of Computational Thinking as an essential cognitive competence, but are these characteristics innate or not? In this work it is investigated whether in children's narratives in the creation of stories emerge patterns correlated to the variables considered essential for the development of computational thinking, based on the learning processes (genetic epistemology) from the cognitive neuroscience and the reflective abstraction for the understanding of the mental processes of children. Mixed methods were used during the process, being experimental part with a sample of twenty-seven individuals, who composed ten narratives. Preliminary results point to the emergence of a pattern, even in the stories that can be classified as "without script", that fit into the possibilities of storytelling by Scratch 2.0 and also in the pillars of Computational Thinking decomposition, pattern, abstraction and algorithm.
\end{abstract}

Resumo. Muito tem sido dito sobre a importância do desenvolvimento do Pensamento Computacional como uma competência cognitiva essencial, mas, seriam essas características inatas ou não? Neste trabalho é investigado se em narrativas infantis, na criação de histórias, emergem padrões correlacionados às variáveis consideradas essenciais para o desenvolvimento do Pensamento Computacional, com base nos processos de aprendizagem (epistemologia genética), a partir da neurociência cognitiva e da abstração reflexionante, para a compreensão dos processos mentais de crianças. Foram utilizados métodos mistos durante o processo, sendo parte experimental, com uma amostra de vinte e sete indivíduos que compuseram dez narrativas. Resultados preliminares apontam para a emergência de um padrão, mesmo nas histórias que podem ser classificadas como "sem roteiro", que se enquadre nas possibilidades de contar história pelo Scratch 2.0 e, também, nos pilares do Pensamento Computacional - decomposição, padrão, abstração e algoritmo.

\section{Introdução}

Pensamento Computacional é um termo que se tornou popular a partir do emblemático discurso de Jeannette Wing (2006), que o defendeu como uma habilidade cognitiva essencial e particular a todos os indivíduos, que lhes possibilitaria resolver problemas. Apesar de ser proferido com intuito de gerar uma injeção de ânimo na área da computação, que na época passava por uma crise, deixava claro que não se tratava somente de decodificar e sim algo muito além disso. A partir de então, muitos têm se 
VII Congresso Brasileiro de Informática na Educação (CBIE 2018)

Anais do XXIX Simpósio Brasileiro de Informática na Educação (SBIE 2018)

voltado à questão de como promover o desenvolvimento do Pensamento Computacional em crianças (Grover \& Pea, 2013)..

Antes da escrita, as formas de registro da humanidade eram as manifestações gráficas e a oralidade (Kress, 2005), dessa forma as tecnologias, registros e estratégias foram passando de geração em geração, permitindo a resolução de problemas e a implementação de inovações, seria então o Pensamento Computacional inato? Assim como as habilidades que permitiram o nascimento da escrita que, para Dehane, foi possível graças à existência de circuitos neurais específicos, uma arquitetura de prérepresentações (Dehaene, 2012). Deste modo, diversas iniciativas têm tido como foco direcionar o "Pensamento Computacional" para "pensar sobre computação", através da seleção de pessoas com habilidades em programação, ou melhor, competência na resolução de problemas por computador (modelagem teórica e codificação), para que possam desenvolver essas habilidades para além das salas de aula (Seehorn et al., 2011). Como bem defende Basawapatna et al. (2011), a visão de que PC trata de se saber programar é reducionista, pois resolver os problemas do mundo real exige mais que isto.

Seria possível identificar nas estruturas narrativas, de crianças, padrões dos conceitos inerentes ao PC? Este trabalho tem como objetivo verificar se existe uma emergência de padrão nas variáveis descritas em narrativas infantis, examinar se existe conexão entre codificação e inferência nas histórias produzidas e, de maneira análoga, julgar se é possível fazer a transposição, quando se usa no processo de criação a ferramenta Scratch.

O restante deste artigo está organizado como segue: na Seção 2 são apresentados alguns trabalhos relacionados da literatura e, em seguida, na Seção 3, a fundamentação teórica, pedagógica e tecnológica necessárias para o entendimento da proposta apresentada na Seção 4. O relato da experimentação realizada e análise dos resultados obtidos são feitos na Seção 5 e, por fim, na Seção 6 são tecidas as considerações finais.

\section{Trabalhos relacionados}

Considerando a ferramenta de programação visual Scratch como um ambiente que incentiva a criatividade e facilita o aprendizado de programação para iniciantes, Wilson e Moffat (2010) aplicaram aulas de programação com Scratch, para crianças com 8 anos, com a finalidade de avaliar benefícios afetivos e cognitivos. As aulas foram voltadas para: introdução ao Scratch, lógica sequencial, estruturas de repetição, estruturas condicionais e coordenação. Uma das vantagens da utilização do Scratch, observada pelos autores, foi tornar a aprendizagem uma experiência positiva e divertida, ao contrário de outras experiências que são consideradas frustrantes pelos alunos. Como benefício afetivo, o Scratch se mostrou motivador e divertido para as crianças, o que resultou em um progresso cognitivo.

Burke e Kafai (2012) realizaram um estudo com alunos do ensino médio, para avaliar a relação existente entre programação e elementos narrativos, por meio da construção de histórias digitais abstraídas de uma oficina de redação. A oficina passou por cinco etapas até chegar ao produto final, sendo: planejamento/redação; elaboraçãoStoryboard; revisão-início da produção; edição-correção e publicação/apresentação. Ao longo do processo, os alunos tiveram aulas de programação e Storytelling, e puderam aplicar tais conceitos nos seus projetos individuais. Como resultados, os autores encontraram padrões entre as histórias, no que diz respeito à escrita do código e, também, 
VII Congresso Brasileiro de Informática na Educação (CBIE 2018)

Anais do XXIX Simpósio Brasileiro de Informática na Educação (SBIE 2018)

a elementos comuns entre narrativas. A aplicação do projeto provou a conexão entre escrita e codificação e incentivou a aprendizagem de programação nas escolas.

Sieckenius et al. (2011) realizaram um estudo sobre a conexão entre representações de linguagem natural e programação. A pesquisa foi realizada com alunos do nono ano do ensino fundamental, utilizando a ferramenta de criação de jogos AgentSheets. Os autores realizaram uma análise semiótica e linguística para comparar a representação de significados entre o jogo produzido pelos alunos e a descrição do jogo em linguagem natural, feita pelos mesmos. Nos projetos finais foram visualizados diferentes níveis de complexidade com relação ao Pensamento Computacional, bem como diferentes níveis de construção textual, porém não existia uma equivalência entre isso, pois um aluno que possuía uma boa narrativa, não necessariamente desenvolvia um bom jogo. Outros resultados apontaram para importantes fatores na aquisição do Pensamento Computacional. Vários estudos já foram realizados nessa vertente, o que comprova a importância e a preocupação existente nos dias atuais quanto a utilização do Pensamento Computacional em processos de aprendizagem.

\section{Pensamento Computacional e a importância das narrativas}

Aprender a codificar o mundo real, em dados legíveis para as máquinas, é uma variável condicionante para se resolver problemas do mundo real, com soluções digitais. Nas décadas de 1950 e 1960 surgiu o conceito de "pensamento algorítmico", que consistia em uma "orientação mental para a formulação de problemas, que convertia uma entrada em uma saída" (Denning, 2009). Em 1975, Laureate Ken Wilson, Nobel da Física, defendeu que a Computação e a Simulação permitiriam fazer Ciência de maneira que até o momento era desconhecida. No começo da década de 1980, Laureate e outros cientistas defendiam que a Computação apresentava solução para grandes problemas, assim a mesma emerge como terceiro pilar da Ciência, e temos a trindade "Teoria, Experimentação e Computação", nessas discussões desponta o que seria chamado de Pensamento Computacional (PC), do inglês "Computational Thinking" (Denning, 2009). Papert e Solomon, em (1971), no artigo "Twenty things to do with a computer", evidenciam algumas ideias que permeiam o PC, mas o termo só aparece em (1980), no livro "Mindstorms: Computers, Children and Powerful ideas" de Seymour Papert.

Apesar do longo histórico, as discussões no entorno do conceito de Pensamento Computacional ganharam forma através das discussões de Jeannette Wing (Wing, 2006), que o define como uma habilidade fundamental para qualquer pessoa, e não somente para um cientista da computação, deixando claro que o pensamento crítico deve ser associado a elementos da computação, e deixa entrever como uma metodologia para resolução de problemas. Smith et al. (2014) definem como os processos de pensamento utilizados na resolução de um problema, cuja representação permite a execução por um agente de processamento de informações. Em (2017), Wing publicou um artigo fazendo um resumo das discussões em torno do sentido e conceito de PC, define que a abstração é uma das principais chaves, fator em que Resnick também acredita (Resnick \& Robinson, 2017). Wing defende ainda que PC permeia todas as Ciências e não somente a Ciência da Computação, abrindo um tópico para falar sobre a importância de se incorporar estes saberes aos processos educativos, para que faça parte da vida dos indivíduos e dela seja indissociado. 
VII Congresso Brasileiro de Informática na Educação (CBIE 2018)

Anais do XXIX Simpósio Brasileiro de Informática na Educação (SBIE 2018)

Existem muitas propostas sobre a fundamentação do Pensamento Computacional, mas, para esta análise, serão utilizados os quatro pilares defendidos pela BBC (2018). O primeiro: decomposição - capacidade de se dividir um problema complexo em problemas menores, facilitando sua resolução. $O$ segundo: padrão - o que permite a verificação aprofundada de um problema menor e a notação de semelhanças que permitirão a automação do processo, ou seja, o que poderia ser replicado. O terceiro: abstração analisar e filtrar o que é realmente importante para a resolução do problema. E o quarto pilar de PC: o algoritmo - o conjunto de passos lógicos necessários para se chegar a resolução do problema.

Desde que se têm registros os homens contam histórias e deixam suas impressões, o que possibilitou a evolução da humanidade, resolvendo problemas conforme lhes era apresentado. Não existe Aprendizagem sem a memória, e a memória da humanidade, por muito tempo, foi passada de geração em geração pela oralidade, por meio de técnicas mnemônicas que hoje ganham explicação através das redes neurais e os mecanismos do sistema nervoso central (Guerra, 2011; Kandel, 2009).

Para Graesser et al.(1980), uma narrativa pode ser a descrição de uma série de ações e eventos, desenvolvidos ao longo do tempo, graças a princípios causais. As narrativas estão carregadas de significado e, através delas, é possível identificar estruturas lógicas essenciais ao Pensamento Computacional. As histórias possuem variáveis de diversos tipos, uma trama desenvolvida a partir das relações estabelecidas pelo tema, noções de estruturas de repetição, condicionais, paralelismo, análise de dados, entre uma série de outros conceitos que foram estendidos para a área da Ciência da Computação, que fazem parte de como se manipula os objetos do mundo real, abstratos ou não. Através de narrativas pode-se avaliar a "inferência", definida por McKonn \& Ratclif (1992) como um fragmento de informação não explícito no texto, é essa variável que define a codificação da relação entre duas instâncias da mesma classe (que dizem respeito ao mesmo conceito).

Pesquisas no cenário cognitivo da compreensão e produção de narrativas têm diversos modelos de análise, sobretudo no que se refere a problemas advindos de síndromes e acidentes que tenham afetado as áreas cerebrais(de Mata, Silva, \& Haase, 2007). A Teoria da Mente acredita que uma das áreas implicadas pode ser a região têmporo-parietal, a mesma relacionada à compreensão e produção de narrativas, que tem com ponto comum a necessidade de organizar a ordem das frases para formação de representações (de Mata et al., 2007; Mar, 2004).

Por outro lado, Jean Piaget (1996) defende as funções simbólicas e suas manifestações, que são formadas a partir da imitação, imagens mentais, jogos simbólicos, desenhos e linguagem, essenciais para a formação do ser humano e amplamente encontradas nas situações em que a criatividade é expressa, inclusive em narrativas (J. Piaget \& Vasques, 1996). A função simbólica é o que permite estabelecer um significado aos objetos (variáveis), por exemplo, quando crianças usam objetos circulares para dizer que estão dirigindo. $\mathrm{O}$ processo se dá de acordo com o desenvolvimento humano, onde se estabelecem camadas de abstração entre as análises realizadas e os significados abstraídos, identificado através da "moral da história", existente ao fim das narrativas, sendo possível afirmar que este é um processo natural.

Pesquisadores afirmam que a criatividade é essencial para o mundo que se descortina a partir do século XXI (Papert, 1980; Resnick \& Robinson, 2017; Wing, 2017). 
$\mathrm{Na}$ criatividade habitam as possibilidades de se resolver problemas, originando a engenhosidade e uma nova forma de enxergar o mundo. Pensando na importância que o protagonismo de ações toma, o Lifelong Kindergarten Group do MIT Media Lab, lança, em 2007, o Scratch, linguagem de programação visual que foi construída para pessoas de diversas idades, com a intenção de facilitar a aprendizagem de programação. O Scratch possibilita aos seus usuários criar seus próprios jogos e histórias animadas, incentiva $o$ pensamento criativo e estimula a aprendizagem de conceitos computacionais. E esses conceitos estão inseridos na habilidade de "pensar computacionalmente".

As narrativas, as possibilidades de entrelaçamento (ideias/concretização) e a ferramenta Scratch encontram um terreno fértil no Construcionismo de Seymour Papert (1980), que defende que a aprendizagem é mais efetiva a partir da ação do indivíduo na construção de suas experiências, em que torna concreto os conceitos até então abstratos, por meio da resolução de problemas.

\section{Proposta}

O método de investigação utilizado foi do tipo hipotético dedutivo, utilizando uma abordagem quase-experimental transversal, com o objetivo de responder as seguintes hipóteses:

Hi: É possível identificar nas estruturas narrativas, de crianças, padrões dos conceitos inerentes ao Pensamento Computacional (PC).

$\mathbf{H}_{\mathbf{j}}$ : As narrativas de histórias infantis, racionalizadas a partir de estruturas do Pensamento Computacional (PC) são executáveis na ferramenta Scratch 2.0.

Foi realizada uma oficina com 27 crianças, com idades entre 10 a 12 anos, sendo 12 meninas e 15 meninos. $78 \%$ das crianças $(21)$ foram selecionadas de uma mesma escola da rede pública de ensino, os outros $22 \%$ são de diferentes escolas, públicas e particulares.

\subsection{Design Experimental (Design e Procedimento)}

A oficina foi estruturada para ter três momentos de interação e promoção da aprendizagem, conforme a figura abaixo (Figura 1):

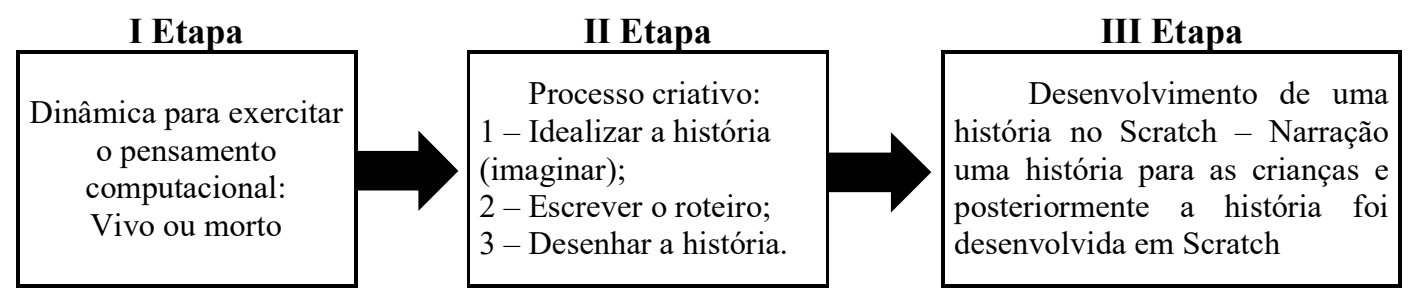

Figura 1. Etapas do processo.

I Etapa: O objetivo foi estabelecer um grau de equidade no estado físico entre as crianças presentes, através do estabelecimento de um exercício (brincadeira) com movimentos corporais, assim quem estivesse muito agitado, poderia ter uma forma de gastar essa energia e quem estivesse mais sonolento (a oficina foi manhã) poderia ser despertado pela produção de serotonina advinda do exercício físico e da ludificação.

Foram explicados os conceitos de computação e de que estes seriam encontrados nas mais variadas tarefas do dia a dia, como na brincadeira de Vivo ou Morto, que poderia ser escrita em pseudocódigo como na Figura 2: 
VII Congresso Brasileiro de Informática na Educação (CBIE 2018)

Anais do XXIX Simpósio Brasileiro de Informática na Educação (SBIE 2018)

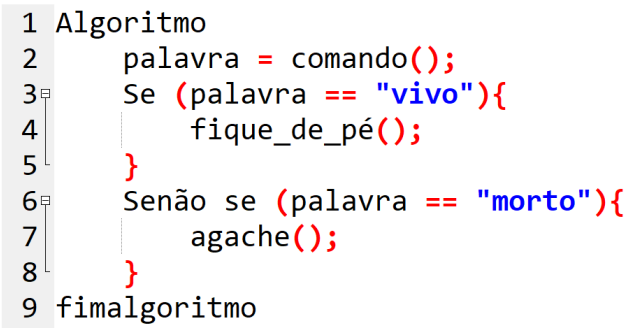

Figura 2. Algoritmo vivo-morto (Fonte: Autores)

O instrutor repassava comandos "vivo" ou "morto", quem errasse o movimento, saia da roda de brincadeiras. A partir dessa atividade, promoveu-se a interação social entre o grupo, que conforme explicitado, nem todos se conheciam e pertenciam ao mesmo grupo (escola). As crianças puderam ver a aplicação da estrutura "se" "senão" "enquanto", mesmo que de forma implícita.

II Etapa: Criando Histórias. O objetivo da ação foi fomentar a criatividade, cujo resultado é a produção de histórias, livres de contextos, produzidas a partir do entrelaçamento de ideias. A sala foi dividida em equipes, 09 equipes com três pessoas em cada uma. As crianças tiveram acesso a um espaço que continha lápis de cor, canetinhas coloridas, papéis coloridos e branco, massa de modelar, cola, tesoura, etc. Assim ela poderia compor o protótipo de sua história conforme a sua criatividade e o que julgasse importante. As etapas descritas abaixo, tiveram uma temporização, conforme descrito e foram divididas da seguinte forma:

II.1. Brainstorm (5min) - Momento para compartilhar ideias, as crianças foram instruídas a discutir e estruturar suas articulações para criação de uma única história, que seria a do grupo, nesse processo, os membros deveriam ser os mediadores do processo (auto mediação).

II.2. Roteirização (10min) - No roteiro é possível analisar a estrutura narrativa e a construção sequencial das ideias, assim como os elementos presentes na narração. As crianças descreveram a história, definiram os personagens, tema e sequência de ações.

II.3. Storyboard (15min) - As crianças elegeram as partes mais importantes de sua história para compor a sequência de ações descritas por meio de desenhos. Em seguida, os grupos compartilharam as suas histórias com os colegas.

III Etapa - Os instrutores contaram uma história para a turma e em seguida, se propuseram a demonstrar, como seria essa produção no ambiente Scratch 2.0.

\section{Resultados e Discussões}

\subsection{As narrativas e o Pensamento Computacional}

As atividades foram realizadas com 27 crianças e divididas em 9 times, com 3 membros. Os times ficaram distribuídos: três times compostos somente por meninos, dois por meninas e quatro times mistos. Entre as equipes mistas, duas com predominância feminina e duas com predominância masculina. 
VII Congresso Brasileiro de Informática na Educação (CBIE 2018)

Anais do XXIX Simpósio Brasileiro de Informática na Educação (SBIE 2018)

Tabela 1. Relação entre os temas, variáveis e elementos do PC.

\begin{tabular}{|c|c|c|c|c|c|c|c|c|c|c|c|c|c|}
\hline \multirow{2}{*}{ Time } & \multicolumn{2}{|c|}{ Sexo } & \multirow{2}{*}{ Tema } & \multirow{2}{*}{$\begin{array}{c}\text { Hist. } \\
\text { Completa }\end{array}$} & \multicolumn{2}{|c|}{ Variáveis } & \multicolumn{3}{|c|}{ Temporalidade } & \multirow{2}{*}{$\begin{array}{r}\text { Decomp } \\
\text { Brain }\end{array}$} & \multirow{2}{*}{$\frac{\text { Padrão }}{\text { orm }}$} & \multirow{2}{*}{$\begin{array}{c}\text { Algoritmo } \\
\text { Roteiro } \\
\end{array}$} & \multirow{2}{*}{$\frac{\text { Abstração }}{\text { StoryBoard }}$} \\
\hline & $\mathbf{M}$ & $\mathbf{F}$ & & & Prot. & Ant. & inicio & meio & fim & & & & \\
\hline 1 & 3 & - & Herois & 1 & 1 & 1 & 1 & 1 & 1 & 1 & 1 & 1 & 1 \\
\hline 2 & 2 & - & Herois & 1 & 1 & 1 & 1 & 1 & 1 & 1 & 1 & 1 & 1 \\
\hline 3 & - & 3 & Herois & 1 & 1 & 1 & 1 & 1 & 1 & 1 & 1 & 1 & 1 \\
\hline 4 & - & 3 & Lenda- moral & 1 & 1 & 1 & 1 & 1 & 1 & 1 & 1 & 1 & 1 \\
\hline 5 & 3 & - & Herois & 1 & 1 & 1 & 1 & 1 & 1 & 1 & 1 & 1 & - \\
\hline 6 & 3 & - & Circo & 1 & 1 & 1 & 1 & 1 & 1 & 1 & 1 & 1 & - \\
\hline 7 & 1 & 2 & Drama & 1 & 1 & - & 1 & 1 & 1 & 1 & 1 & 1 & 1 \\
\hline 8 & 1 & 2 & Lenda- moral & 1 & 1 & 1 & 1 & 1 & 1 & 1 & 1 & 1 & - \\
\hline 9 & 2 & 1 & Herois & 1 & 1 & 1 & 1 & 1 & 1 & 1 & 1 & 1 & - \\
\hline
\end{tabular}

A Tabela 1 apresenta a desenvoltura dos grupos em relação à criação do roteiro e do Storyboard, e a facilidade em identificar na narrativa o início, o meio e o fim, bem como os quatro pilares do Pensamento Computacional, analisados pela fase de produção das histórias. Considerou-se a variável 1 para as tarefas completadas e 0 para as tarefas não finalizados, como demonstrado, o fato de não ter cumprido com o que se pré-define como Storyboard, não quer dizer que não exista história as equipes foram capazes de narrar uma história com todos os elementos presentes, mesmo que não explicitamente.

Dentre as histórias analisadas, $71,4 \%$ se referem a heróis, $28,6 \%$ são histórias com função moral muito fortes e remontam lendas, 14,3\% falam de um conteúdo diferenciado, aventura em um circo e 1 história representando 14,3\% um drama vivido por um garoto que após uma jornada, vai a óbito. O que permite inferir que $71,4 \%$ as histórias, tem forte componente tecnológico digital, bem como superpoderes.

$\mathrm{Na}$ Tabela 2, é possível verificar a análise entre os pilares do Pensamento Computacional e as atividades realizadas durante a oficina:

Tabela 2. Correlação das atividades com os pilares do Pensamento Computacional.

Pensamento

Computacional

\begin{tabular}{|c|c|}
\hline Decomposição & $\begin{array}{l}\text { Para desenhar o Storyboard as crianças faziam "quadrinhos" no papel, para } \\
\text { desenhar os acontecimentos em ordem cronológica. Ao decompor a história em } \\
\text { diversos quadros, as crianças tornaram mais fácil o trabalho de transpor a história } \\
\text { (escrita) para o desenho }\end{array}$ \\
\hline $\begin{array}{l}\text { Reconhecimento de } \\
\text { padrões }\end{array}$ & $\begin{array}{l}\text { Após o processo de separar por quadros, a criança pôde reaproveitar o desenho do } \\
\text { personagem para diversos quadros. Ou seja, ao reconhecer os padrões entre frases } \\
\text { da história, foi possível desenhar objetos baseados em outros já existentes, } \\
\text { facilitando o processo }\end{array}$ \\
\hline Abstração & $\begin{array}{l}\text { No processo de construção dos desenhos, as crianças selecionam os elementos } \\
\text { mais importantes da história para desenhar, como por exemplo, personagens e } \\
\text { cenários. }\end{array}$ \\
\hline Algoritmo & $\begin{array}{l}\text { A história e o Storyboard são algoritmos, pois tem início, meio e fim. Além das } \\
\text { estruturas lógicas encontradas dentro da história. }\end{array}$ \\
\hline
\end{tabular}

Algoritmos são sequências lógicas com o objetivo de solucionar um problema. Uma história segue uma ordem, ou seja, a lógica está presente no momento de escrever uma história. Como início, meio e fim, em que um primeiro evento possui um problema ou evento desencadeador da ação dos atores, as atividades do protagonista geram com isso consequências, o cenário define as circunstâncias físicas e temporais da ação. Em suma há uma atividade inicial que direciona o personagem para um comportamento para resolver um problema e a consequência desse ato, resolve, ou não o problema proposto. Em uma análise da produção das histórias, todos os elementos listados foram localizados nas narrativas: 
VII Congresso Brasileiro de Informática na Educação (CBIE 2018)

Anais do XXIX Simpósio Brasileiro de Informática na Educação (SBIE 2018)

Tabela 3. Emergência das estruturas lógicas nas histórias.

\begin{tabular}{lll}
\hline \multicolumn{1}{c}{ Estruturas Logicas } & \multicolumn{1}{c}{ Tipo } & \multicolumn{1}{c}{ Localização nas histórias } \\
\hline Variáveis & Diversos & Personagens \\
\hline Definição de Variáveis & Nomeação & Atrib.nomes \\
\hline Estruturas Condicionais & Se, senão, senão se & Relações entre atores \\
\hline Disjunção & Ou & Relação entre atores e ambiente \\
\hline Conjunção & $\mathrm{E}$ & Relação entre atores e ambiente \\
\hline Repetição & $\begin{array}{l}\text { Enquanto, repita n vezes, } \\
\text { repita sempre }\end{array}$ & Relação entre atores e ambiente \\
\hline
\end{tabular}

\subsection{Narrativas e o Scratch 2.0}

Antes da aplicação da oficina, foram estipulados os blocos de programação do Scratch a serem utilizados no processo de repassar a história para o ambiente digital, são eles: Eventos, Movimento, Aparência, Controle e Sons, pelas possibilidades que poderiam proporcionar no que diz respeito a produção de Histórias

Tabela 4. Relação estruturas Scratch vs Execução da história.

\begin{tabular}{ll}
\hline Estruturas & \multicolumn{1}{c}{ Ações desencadeadas } \\
\hline Eventos & Troca de cenário, interação entre personagens (por meio do bloco de envio de mensagens). \\
\hline Movimento & Movimentação do personagem ou de quaisquer objetos pelo cenário. \\
\hline Aparência & Comportamento do personagem (fala, pensamento, simulação de caminhada). \\
\hline Controle & $\begin{array}{l}\text { Repetição de ações dos personagens, influência de uma ação em outra, por exemplo: "as } \\
\text { maçãs irão cair se o rei balançar a árvore" - se rei tocando em árvore então a maçã desliza } \\
\text { até o chão (estruturas condicionais). }\end{array}$ \\
\hline Sons & $\begin{array}{l}\text { Para tornar a história mais interessante podem ser adicionados sons, por exemplo, se existe } \\
\text { um personagem gato, ele pode emitir o som de miado. }\end{array}$ \\
\hline
\end{tabular}

Conforme a Tabela 5, todas as histórias propostas pelas crianças são passíveis de execução em Scratch 2.0, bem como utilizariam todas as estruturas descritas na tabela abaixo:

Tabela 5. Blocos e trabalhos desenvolvidos.

\begin{tabular}{lccccccccc}
\hline \multirow{2}{*}{ Blocos } & \multicolumn{10}{c}{ Trabalhos a serem des envolvidos } \\
\cline { 2 - 10 } & Equipe 1 & Equipe 2 & Equipe 3 & Equipe 4 & Equipe 5 & Equipe 6 & Equipe 7 & Equipe 8 & Equipe 9 \\
\hline Eventos & 1 & 1 & 1 & 1 & 1 & 1 & 1 & 1 \\
\hline Movimento & 1 & 1 & 1 & 1 & 1 & 1 & 1 & 1 & 1 \\
\hline Aparência & 1 & 1 & 1 & 1 & 1 & 1 & 1 & 1 & 1 \\
\hline Controle & 1 & 1 & 1 & 1 & 1 & 1 & 1 & 1 \\
\hline Sons & - & - & - & - & - & - & - & - \\
\hline
\end{tabular}

Como exemplo prático, podemos citar uma das histórias produzida por um grupo de crianças, intitulada de Super jovem, que narra a história de vida de um garoto que cresceu em um orfanato e depois faz descobertas importantes sobre seu passado. A história possui cenário, personagens e linha temporal. Tal história pode ser narrada usando Scratch,pela produção de animações. O Storyboard da equipe pode ser observado na figura3. Em Super jovem, do primeiro quadro do Storyboard é possível extrair a ambientação do cenário onde a história se inicia (um orfanato), identificar a representação do personagem, que inicialmente é uma criança, a narrativa permite inferir a movimentação e entrelaçamento de personagens, como variáveis no enredo. A criança descobre que seus pais foram mortos e, baseado nessa descoberta de que ele não fora 
abandonado e sim vítima de uma injustiça, ele desenvolve superpoderes e luta com vários inimigos.

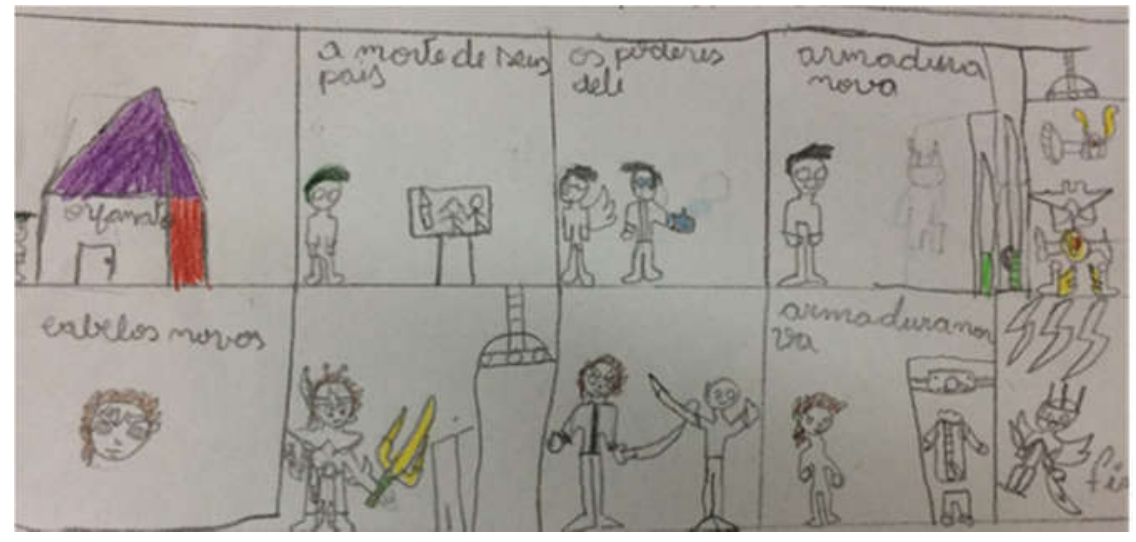

Figura 3. Storyboard da história Super jovem, feito por grupo de alunos na oficina apresentada.

Ao analisarmos a representação dessas lutas no Storyboard, é possível perceber padrões como: $S E$ um inimigo aparecer, o herói saca a espada; $S E$ inimigo morrer, o herói guarda a espada. O grupo, ao realizar a ideação da história decompôs o enredo, organizou de forma padronizada, abstraiu a exposição da informação e seguiu com a narrativa de forma algorítmica. Todos os passos citados, são passíveis de execução em Scratch, como pode ser observado na plataforma compartilhada da linguagem nos trabalhos de outros usuários.

As hipóteses Hi e Hj se confirmam, conforme os dados apresentados, para esta experiência em questão. Podemos citar com ameaças a validade, a amostra reduzida, indicando para teste futuros, replicar as atividades com a finalidade de identificar as mesmas ou outras variáveis com o mesmo efeito conclusivo.

\section{Considerações finais}

Pensamento Computacional tem sido um tema muito discutido, o que confirma a importância que a Ciência vem dando aos processos de desenvolvimento humano com vistas na resolução de problemas do mundo real e na forma como isso poderia ser realizado. Os processos criativos devem ser priorizados na formação dos indivíduos e a análise realizada indicou que mesmo não executando de fato a programação de suas histórias, as estruturas lógicas que permitem o encadeamento de ideias estavam presentes, sendo possível executar uma animação digital com a linguagem de programação Scratch disponível.

Como extensão do trabalho, pretende-se replicar a atividade com uma amostra maior, com uma maior diversidade de faixas etárias e etnias envolvidas, tendo como objetivo testar a hipótese, bem como localizar informações sobre o processo de desenvolvimento do Pensamento Computacional.

\section{Referências}

Basawapatna, A., Koh, K. H., Repenning, A., Webb, D. C., \& Marshall, K. S. (2011). Recognizing computational thinking patterns. Proceedings of the 42nd ACM Technical Symposium on Computer Science Education.

BBC. (2018). Introduction to computational thinking. Retrieved July 9, 2018, from https://www.bbc.com/education/guides/zp92mp3/revision/1 
VII Congresso Brasileiro de Informática na Educação (CBIE 2018)

Anais do XXIX Simpósio Brasileiro de Informática na Educação (SBIE 2018)

Burke, Q., \& Kafai, Y. B. (2012). The writers' workshop for youth programmers: digital storytelling with scratch in middle school classrooms. Proceedings of the 43rd ACM Technical Symposium on Computer Science Education.

de Mata, F. G., Silva, J. B. L., \& Haase, V. G. (2007). Narrativas: abordagens cognitivas e neuropsicológicas da análise da produção e compreensão. Mosaico: estudos em psicologia, 1(1).

Dehaene, S. (2012). Os Neurônios Da Leitura. Porto Alegre: ARTMED.

Denning, P. J. (2009). The profession of IT Beyond computational thinking. Communications of the ACM, 52(6), 28-30.

Graesser, A. C., Hauft-Smith, K., Cohen, A. D., \& Pyles, L. D. (1980). Advanced outlines, familiarity, and text genre on retention of prose. The Journal of experimental education, 48(4), 281-290.

Grover, S., \& Pea, R. (2013). Computational thinking in K-12: A review of the state of the field. Educational Researcher, 42(1), 38-43.

Guerra, L. B. (2011). O diálogo entre a neurociência e a educação: da euforia aos desafios e possibilidades. Revista Interlocução, 4(4), 3-12.

Kandel, E. R. (2009). Em busca da memória: o nascimento de uma nova ciência da mente: Companhia das Letras.

Kress, G. (2005). Before Writing: Rethinking the Paths to Literacy: Taylor \& Francis.

Le Goff, J. (2017). MEMÓRIA. História e memória.

Mar, R. A. (2004). The neuropsychology of narrative: Story comprehension, story production and their interrelation. Neuropsychologia, 42(10), 1414-1434.

McKoon, G., \& Ratcliff, R. (1992). Inference during reading. Psychological review, 99(3), 440.

Papert, S. (1980). Mindstorms: Children, computers, and powerful ideas: Basic Books, .

Papert, S., \& Solomon, C. (1971). Twenty things to do with a computer.

Piaget, J., Inhelder, B., Sinclair, H., \& da Rocha Salles, A. (1972). Memória e inteligência: Ed. Artenova; Ed. Universidade de Brasília.

Piaget, J., \& Vasques, R. A. (1996). A construção do real na criança: Editora Atica.

Resnick, M., \& Robinson, K. (2017). Lifelong Kindergarten: Cultivating Creativity Through Projects, Passion, Peers, and Play: MIT Press.

Seehorn, D., Carey, S., Fuschetto, B., Lee, I., Moix, D., O'Grady-Cunniff, D., . . . Verno, A. (2011). CSTA K--12 Computer Science Standards: Revised 2011.

Smith, N., Sutcliffe, C., \& Sandvik, L. (2014). Code club: bringing programming to UK primary schools through scratch. Paper presented at the Proceedings of the 45th ACM technical symposium on Computer science education.

Souza, C. S., Garcia, A. C. B., Slaviero, C., Pinto, H., \& Repenning, A. (2011). Semiotic traces of computational thinking acquisition. Paper presented at the International Symposium on End User Development.

Wilson, A., \& Moffat, D. C. (2010). Evaluating Scratch to introduce younger schoolchildren to programming. Proceedings of the 22nd Annual Psychology of Programming Interest Group (Univ. Carlos III de Madrid, Leganés, Spain.

Wing, J. (2006). Computational thinking. Communications of the ACM, 49(3), 33-35.

Wing, J. (2017). Computational thinking's influence on research and education for all. Italian Journal of Educational Technology, 25(2), 7-14. 